\title{
Efficient Low-Overhead Channel Estimation for 5G Lens Based Millimeter-Wave Massive MIMO Systems
}

\author{
Imran Khan* \\ Department of Electrical Engineering, University of Engineering \& Technology, Peshawar 814, Pakistan
}

Received: 25 August 2017; Accepted: 13 February 2018; Published: 08 May 2018

\begin{abstract}
Beamspace MIMO performs beam-selection which can substantially reduce the number of power-consuming radio frequency (RF) chains without perceptible performance deterioration. However, for capacityapproaching performance, accurate information of the beamspace-channel of large-size is required for beamselection, which is contesting in case of little number of RF-chains. To overcome such problem, I proposed an efficient support-detection (SD) algorithm for channel-estimation with low pilot-overhead and short number of RF chains. The key idea of SD-algorithm is to divide the whole issue of beamspace channel-estimation into a series of sub-issues, where each of them considers only one sparse channel-component. The support of each channel component is detected reliably by deploying the sparse structure attributes of the beamspace-channel. The effect of this channel-component is eliminated from the whole channel-estimation issue. Thus, the sparse beamspace-channel can be estimated with low pilot-overhead. Simulation Results shows that the proposed schemes perform much better than the conventional compressed-sensing (CS) schemes.
\end{abstract}

Index Terms: mmWave; Beamspace; SD; Pilot; RF chain.

(C) 2018 Published by MECS Publisher. Selection and/or peer review under responsibility of the Research Association of Modern Education and Computer Science

\section{Introduction}

Millimeter-wave (mmWave) massive MIMO is deemed to be a potential technology for future 5G wireless communication systems due to its significant performance metrics such as higher data-rate, wider bandwidth (BW), enhanced energy-efficiency (EE) and spectral-efficiency (SE) respectively. Due to such remarkable features, it has attracted much research interests from various communities. As hundreds of antennas at mmWave frequencies are deployed which results higher number of RF-chains requirement, which increases the energy consumption and implementation cost. The practical realization of mmWave massive MIMO is not an easy task in practice. It is due to the fact that, each antenna requires its own dedicated single RF-chain, which

\footnotetext{
* Corresponding author.

E-mail address: imran_khan@uetpeshawar.edu.pk
} 
consists of digital-to-analog (DAC) converter, low-noise amplifier (LNA), up-converter etc. that consume more energy, gets more complex and takes much hardware budget per device [1]. The energy consumption, complexity and cost increases with increasing the number of antennas. It is the major concerned issue in this work. Also, the energy consumption is greater at mmWave-frequencies i.e. $30-300 \mathrm{GHz}$ as compare to $4 \mathrm{G}$ cellular frequency. To understand it further, consider the example where each RF-chain consume $200 \mathrm{~mW}$ of energy at the mmWave frequency that is much higher than the RF-chain which operates at cellular frequencies of $6 \mathrm{GHz}$ and consume $25 \mathrm{~mW}$ of energy. So, the ratio $200 \mathrm{~mW}: 25 \mathrm{~mW}$ results in eight-times more energy consumption in mmWave system. Moreover, if a base-station (BS) with 250-antennas operate at mmWave frequencies, the required $260 \mathrm{RF}$-chains will consume $52 \mathrm{~W}$ of energy which is much larger than the $4 \mathrm{G}$ cellular system which consume $6.5 \mathrm{~W}$ respectively. To decrease the required number of power-dissipating RFchains in the proposed system, the idea of beamspace-MIMO is recently proposed in the research literature [2]. The beamspace MIMO uses the lens-antenna array in place of the conventional electromagnetic (EM) antenna array to convert the conventional spatial-channel into beamspace-channel by focusing the signals beams (energy) from different channel directions on different antenna elements.

The characteristics of mmWave channels has limited scattering which results in much limited number of sparse propagation paths that occupy a small number of beams. Thus, the beamspace channel of mmWave exhibit sparse structure, and I can choose such small number of prevailing beams to substantially decrease the MIMO system channel-dimension and the required number of RF-chains through beam selection process without noticeable performance deterioration. The beam-selection process imposes a condition on the BS to obtain the large-size beamspace-channel information, which is tedious task in case of little number of RFchains. To solve this issue, recent research proposed some sophisticated compressed-sensing (CS) algorithms to exploit the mmWave channels angle-domain sparsity in order to estimate the mmWave massive MIMO largesize channels efficiently [3]- [5]. However, these algorithms are based on hybrid precoding. The beamspace channel estimation issue for MIMO system is not well addressed in the literature.

In this paper, I propose a novel approach of reducing the number of RF-chains, increasing the channel estimation efficiency by using low pilot-overhead and enhancing the sum-rate by deploying a reliable supportdetection (SD) algorithm. The basic principle of SD is to divide the whole task of beamspace-channel into a series of sub-tasks, in which each sub-task considers only one sparse-channel component, i.e. a channel-vector which contain the information of a particular propagation-path. The support or nonzero elements set index of sparse-vector is detected for each channel-component in compliance to the strongest-element position. After this, the effect of this channel-component is eradicated from the entire beamspace-channel estimation task and the support-element of the subsequent channel-component is detected using the same procedure. After detecting all supports of channel-components, the sparse beamspace channels nonzero-elements can be estimated with low pilot-overhead. The results are compared with the classical compressed-sensing (CS) algorithms for performance evaluation.

The remaining paper is organized as follows. Section II describes the literature review of the proposed research. The propose system model is explained with mathematical modeling in Section III. The simulation results are provided in Section IV. Section V concludes the paper.

\section{Literature Review}

The drastic rise of smartphones, notebooks, tablets devices and mobile data communication are producing extraordinary challenge for the cellular service providers and researchers [6]. In one estimate, by 2020 the number of new devices beyond the smartphones and tablets connected such as the IoT-sensors, cloud-devices will be 50 billion [7]. Current cellular service providers try to deliver high quality of service (QoS), low-latency, high data-rate multimedia applications for the wireless devices, but their bandwidth (BW) is scarce and limited. The current 4G technology is unable to meet the 1000-fold increase in data-traffic which is predicted for 5G wireless systems that demands for novel wider spectrum bands and devices with greater spectral efficiency.

The current $4 \mathrm{G}$ cellular system confronts severe spectrum shortage for which the mmWave spectrum of 30$300 \mathrm{GHz}$ is attracting much interest as it is a promising technology for the future $5 \mathrm{G}$ microcell and picocell wireless networks. As the success of cellular mobile technologies is evident, which demands for more boosting 
mobile data traffic, the universal mobile telecommunication system (UMTS) forecast that by 2020, the daily mobile traffic will exceed $800 \mathrm{MB} /$ user which leads to $130 \times 10^{18}$ bits data per year for operators. The worldwide shortage of BW has motivated the researchers to utilize the underutilized millimeter-wave (mmWave) spectrum for $5 \mathrm{G}$ wireless communication networks. The proposed mmWave spectrum is 200 -times more than the current cellular spectrum allocation. It enables the deployment of very high-dimensional antenna-arrays to further increase the gain by spatial-multiplexing and beamforming. As the mmWave has very shorter wavelengths, so it can be used in low-power CMOS RF hardware which is cost-effective and easy to implement. The combination of MIMO with mmWave has got much attention from academic and industry because the mmWave offer more than $2 \mathrm{GHz} \mathrm{BW}$ for cellular system which is much greater than the $4 \mathrm{G}$ cellular system BW of $20 \mathrm{MHz}$. Moreover, the mmWave has short-wavelengths which permit a large antenna-array to be assembled in a small area, thus allowing to obtain very high-gain, high-directivity, electrically-steerable and high degrees of freedom (DoF) [8]. To utilize the proposed mmWave BW, beamspace MIMO based on lensantenna array is proposed, which utilizes its BW as well as provide efficient channel estimation and enhances the energy efficiency (EE).

The integration of millimeter-wave and massive MIMO is considered to be the potential promising technology for the $5 \mathrm{G}$ wireless communication systems due to its high spectral efficiency, wider bandwidth availability and improved energy efficient [9]. The mmWave bandwidth is from $30-300 \mathrm{GHz}$ which is underutilized, and it is utilized by the incorporation of massive MIMO. Lens-based mmWave massive MIMO technology can substantially reduce the required number of RF chains without any noticeable performance degradation. It solves the problem of traditional MIMO systems in which each antenna requires its own dedicated RF chain which results in high energy consumption and increased complexity due to the increased number of RF chains deployment. The main function of the lens-antenna array is the transformation of the conventional spatial channel into beamspace channel which has sparse structure, which is performed by focusing beams from different directions on different antennas [10]. The sparse structure of the mmWave beamspace channel is due the limited number of effective propagation paths, which is utilized for efficient channel estimation with low pilot overhead. Moreover, it facilitates to select few significant beams to substantially decrease the number of RF chains as well as the MIMO system dimension respectively [11]. The calculation of high-dimension beamspace channel information is challenging for the proposed system as it deploys limited number of RF chains in practice [12]. Recent research proposed two types technique to solve this problem [13]- [15]. The first type is based on the principle to reduce the beamspace channel dimension effectively in order to simplify the problem of channel estimation [13], [15]. Particularly, the beam training determines the selected beams to reduce the dimension of beamspace channel. After this, the least-square (LS) algorithm can be used to estimate the dimension-reduced beamspace channel with low pilot-overhead respectively. On the other hand, the second type algorithm utilizes the beamspace channel sparsity to further decreases the pilot-overhead. It is based on compressive-sensing (CS) theory which accurately estimate the support-vector of the sparse beamspace channel. The support means the set of nonzero elements in the sparse channel vector. The existing algorithms for beamspace channel are based on the 2D channel model, which can only deploy the horizontal degrees of freedom (DoF). On the other hand, the proposed 3D channel model for beamspace channel estimation is more generic which can deploy both horizontal as well as vertical DoFs, which is not yet addressed in the literature.

Channel-estimation is a difficult and challenging problem for mmWave massive MIMO which deploys hybrid-precoding, that faces problem as the number of antennas are huge than RF-chains. Conventional compressed-sensing (CS) algorithms for channel estimation suffer due to channel angle quantization high resolution loss. Several novel algorithms are recently proposed for channel estimation in mmWave massive MIMO which deploy hybrid precoding [16]-[20]. Particularly [16] and [20] proposed channel estimation algorithms which are based of adaptive codebook, in which the transmitter $\left(T_{x}\right)$ and receiver $\left(R_{x}\right)$ search for the most dominant beam-pair. The search is performed by adjusting the pre-defined precoding and combining codebooks. The problem with this algorithm is that the resolution of channel estimation is bounded by the codebook size constraint. 
None of the existing schemes offer spectral and reduced RF based efficient channel estimation. Therefore, I proposed a support detection (SD) algorithm for the lens-based beamspace channel-estimation that is performing much better than the existing schemes with more reliability.

\section{System Model}

A typical system of mmWave massive MIMO is considered with $\mathrm{N}$ number of BS-antennas, $\mathrm{K}$ number of single antenna users and $\mathrm{N}_{\mathrm{RF}}$ number of RF-chains respectively. The time-division duplexing (TDD) mode of operation is used for the proposed system. First, the conventional-MIMO system is considered and then it is converted into beamspace MIMO system respectively. Both conventional and beamspace MIMO systems model are shown in Figure 1.

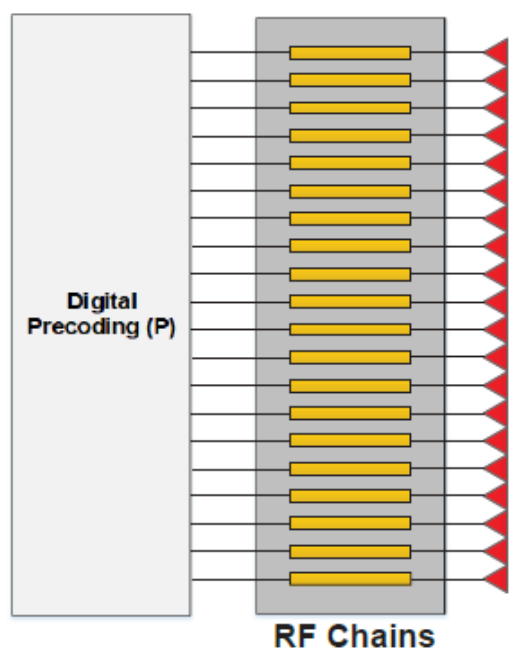

(a)

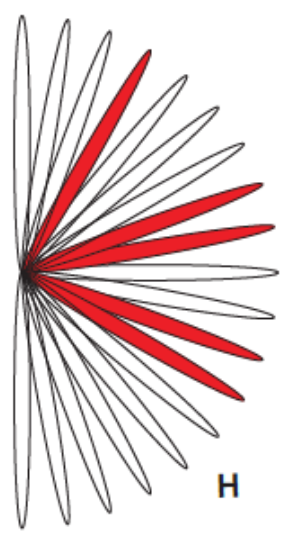

$\underbrace{\square . . .}_{\text {User } 1}$
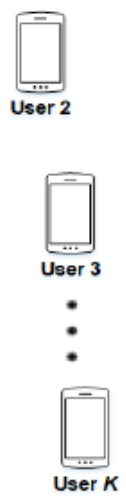

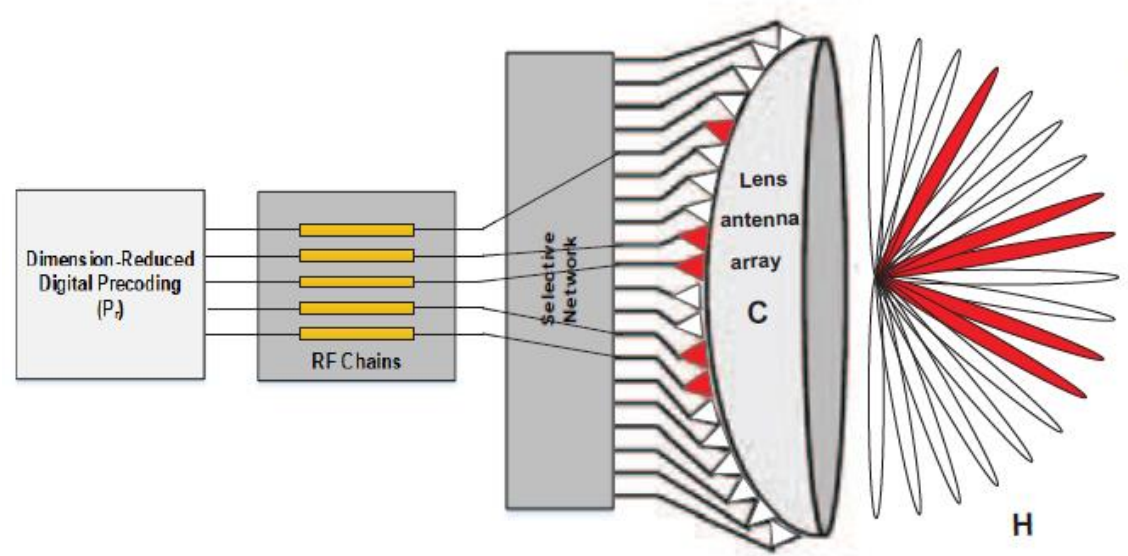

(b)

$\underset{\text { User } 1}{\square . .1}$
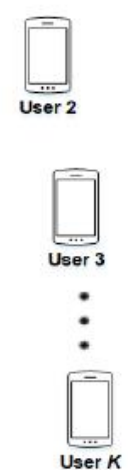

Fig.1. (a) Conventional MIMO; (b) Beamspace-MIMO. 
The downlink (DL) received signal-vector for the conventional MIMO for all K-users is expressed as:

$$
y^{D L}=H^{H} P s+n
$$

Where $y^{D L}$ is the $K x 1$ received signal-vector, $H^{H} \in \mathbb{C}^{K x N}$ is the DL channel-matrix, $H=\left[h_{1}, h_{2}, h_{3}, \ldots, h_{K}\right]$ is the UL channel-matrix, $h_{k}$ is the channel-vector between the BS and $k$ th-user, $s$ is the signal-vector of $K x 1$ dimension for all $K$-users, with normalized power of $I_{K}, P$ is the precoding-matrix of order $N x K, n$ is the noise-vector of size $K x 1$ which follows the complex Gaussian distribution $C N\left(0, \sigma_{D L}^{2} I_{K}\right)$, where $\sigma_{D L}^{2}$ is the DL noise-power.

\subsection{Millimeter-Wave channel model}

The Saleh Valenzuela channel-model is deployed for the proposed mmWave channel-model which is mostly used for this purpose. The conventional spatial-domain channel is then expressed by:

$$
h_{k}=\sqrt{\frac{N}{L+1}} \sum_{i=0}^{L} \beta_{k}^{i} a\left(\psi_{k}^{i}\right)=\sqrt{\frac{N}{L+1}} \sum_{i=0}^{L} c_{i}
$$

Where, $c_{0}=\beta_{k}^{0} a\left(\psi_{k}^{0}\right)$ is the line-of-sight ( $\left.\operatorname{LoS}\right)$ part of the $k$ th-user, $\beta_{k}^{0}$ is the complex-gain of the channel, $\psi_{k}^{0}$ is the spatial-direction of the propagation path, $c_{i}=\beta_{k}^{i} a\left(\psi_{k}^{i}\right)$ is the $i$ th non-line-of-sight (NLoS)component of the $k$ th-user for $1 \leq i \leq L$, and $L$ is the whole number of NLoS-components which is determined from measurement of channel vectors, $a(\psi)$ is the array steering-vector of order $N x 1$ which is obtained for the typical N-uniform linear-array (ULA) antennas as follows:

$$
a(\psi)=\frac{1}{\sqrt{N}}\left[e^{-j 2 \pi \psi m}\right]_{m \in !(N)}
$$

Where $\mathrm{I}(N)=\left[l-\frac{\{N-1\}}{2}, l=0,1,2, \ldots, N-1\right]$ is set of the indices of symmetric-set centered around-zero and $\psi$ is the spatial-direction which is obtained by:

$$
\psi \triangleq \frac{d}{\lambda} \sin \theta
$$

Where $\theta$ is the signal physical direction, $\lambda$ is the wavelength of the signal, $d$ is the separation or spacing of antenna elements which satisfy the half-wave condition $\left(d=\frac{\lambda}{2}\right)$ at the proposed mmWave frequencies.

\subsection{Transformation to Beamspace MIMO}

The conventional spatial-domain channel of (2) is converted into the beamspace-channel by using a sophisticated lens-antenna array as shown in Figure 1(b). The lens-antennas array works as a spatial discrete Fourier transform (DFT) matrix $\mathbb{\Gamma}$, which consists of N-orthogonal beams array steering-vectors $a(\psi)$ that covers the entire angle-space which is given by:

$$
\mathbb{\Gamma}=\left[a\left(\bar{\psi}_{1}\right), a\left(\bar{\psi}_{2}\right), a\left(\bar{\psi}_{3}\right), \ldots, a\left(\bar{\psi}_{N}\right)\right]^{H}
$$

Where $\bar{\psi}_{N}=\frac{1}{N}\left(n-\frac{N+1}{2}\right), n=1,2,3 \ldots, N$.

Now, by supposing that the number of users is equal to the RF-chains i.e. $N_{R F}=K$, the beamspace MIMO channel is then given by: 


$$
\hat{y}^{D L}=H^{H} \mathbb{\Gamma}^{H} S P_{r} s+n=\widehat{H}^{H} S P_{r} s+n
$$

Where, $\hat{y}^{D L}$ is DL received-vector in beampsace, $\widehat{H}^{H}=H^{H} \mathbb{\Gamma}^{H}=(\mathbb{\Gamma} H)^{H}$ is the DL channel-matrix of beamspace, and its N-columns maps to N-orthogonal beams, $S \in\{0,1\}$ is the selecting-matrix of $N x K$ dimension. It means that the selected beam at the corresponding user will be 1 and other will be $0, P_{r}$ is the precoding-matrix of reduced-dimension whose size is $K x K$.

It is important to note that the beamspace channel-matrix in (6) exhibit sparse-structure because of the minute number of scattering paths which are dominant in the mmWave propagation environments. Thus, only such small sparse beams can be selected according to the beamspace-channel which can substantially decrease the pilot-overhead due to the reduced dimenstion of the channel without any perceptible performance deterioration. Therefore, due to such little number of sparse channel paths, a smaller size digital-precoder is required for generating the precoding-matrix $\left(P_{r}\right)$. This mechanism also reduces the number of RF-chains for operation.

\subsection{Beamspace Channel Estimation}

Figure 1(b) is considered to determine the beamspace channel-estimation. The entire process is decomposed into three main steps in which the pilot-transmission is first determined. Second, the beamspace measurement is performed and finally, the proposed SD-algorithm estimate the beamspace channel with low pilot-overhead and little number of RF-chains.

Pilot Transmission: For channel estimation, the users are required to transmit their known pilot-sequences to the BS over $T$-slots where, each user transmit one pilot-signal in each time-slot. The beamspace channel is assumed to remain the same within such $T$-slots interval. The $T$-slots are divided into $M$-blocks where each block contains K-time slots, therefore, $T=M K$. The pilot-matrix $\Phi_{m}$ of the $m$ th-block of size $K x K$ is defined which consists of $K$-mutually orthogonal pilot-signals that are transmitted over $K$-slots by the $K$-user respectively. It is also clear that due to orthogonality:

$$
\Phi_{m} \Phi_{m}{ }^{H}=\Phi_{m}{ }^{H} \Phi_{m}=I_{K}
$$

Now, in the proposed TDD system using the channel-reciprocity theorem from [13], the UL received-signal at the $\mathrm{BS}$ in the $m$ th-block is expressed by:

$$
\hat{y}_{m}^{U L}=\Gamma H \Phi_{m}+\mathrm{n}_{m}=\widehat{H} \Phi_{m}+n_{m}
$$

Where $m=1,2,3, \ldots, M, \hat{y}_{m}^{U L}$ is the UL received-signal matrix of order $N x K, \mathrm{n}_{m}$ is the noise-signal matrix of order $N x K$ whose elements are independently and identically distributed (i.i.d) and follow complex Gaussian distribution with zero-mean and $\sigma_{U L}^{2}$ variance which is the UL noise-power.

Beamspace channel measurement: The BS deploy a combiner $\mathrm{C}_{m}$ of size $K x N$ to combine the UL receivedsignal of (8). The $\mathcal{R}_{m}$ in the baseband of of order $K x K$ which is sampled by $N_{R F}=K$ is given by:

$$
\mathcal{R}_{m}=\mathrm{C}_{m} \hat{y}_{m}^{U L}=\mathrm{C}_{m} \widehat{H} \Phi_{m}+\mathrm{C}_{m} n_{m}
$$

The measurement-signal matrix $Y_{m}$ of the beamspace channel-matrix of order $K x K$ is determined by multiplying (9) with the known pilot-signal $\Phi_{m}^{H}$ which is given as follows:

$$
Y_{m}=\mathcal{R}_{m} \Phi_{m}^{H}=\mathrm{C}_{m} \widehat{H}+\mathrm{n}_{m}^{e f f}
$$

Where $\mathrm{n}_{m}^{e f f}=\mathrm{C}_{m} n_{m} \Phi_{m}^{H}$ is the effective noise-signal matrix. 
Here, the main concern is to estimate the $k$ th-user beamspace-channel $\hat{h}_{k}$ and the rest of the users beamspace channel is determined in the same manner in order to get the entire beamspace channel $\widehat{H}$ respectively. The $T \times 1$ measurement-vector after $M$-blocks transmission of pilot-signal can be calculated for the $k$ th-user having beamspace channel-vector $\hat{h}_{k}$ is given by:

$$
\left[\bar{y}_{k}\right]_{T \times 1}=\left[\begin{array}{c}
y_{1, k} \\
y_{2, k} \\
y_{3, k} \\
\vdots \\
y_{M, k}
\end{array}\right]=\left[\begin{array}{c}
C_{1} \\
C_{2} \\
C_{3} \\
\vdots \\
C_{M}
\end{array}\right] \hat{h}_{k}+\left[\begin{array}{c}
\mathrm{n}_{1, k}^{\text {eff }} \\
\mathrm{n}_{2, k}^{\text {eff }} \\
\mathrm{n}_{3, k}^{e f f} \\
\vdots \\
\mathrm{n}_{M, k}^{e f f}
\end{array}\right] \triangleq[\bar{C}]_{T \times N} \hat{h}_{k}+\left[\bar{n}_{k}\right]_{T \times 1}
$$

Where $y_{m, k}, \hat{h}_{k}$ and $\mathrm{n}_{m, k}^{e f f}$ are the kth-columns of the corresponding $Y_{m}, \widehat{H}$ and $\mathrm{n}_{m}^{\text {eff }}$.

The main goal is to recover $\hat{h}_{k}$ reliably with pilot-overhead $T$ as low as possible which is based on $\bar{y}_{k}$ measurements. If we use the conventional selecting-network (CSN) of Figure 1(b) directly to design a combiner $\bar{C}$ for the BS, each row of it will have only one nonzero-element. Furthermore, to assure that $\bar{y}_{k}$ measurements encompass the entire beamspace channel $\hat{h}_{k}$ information, the overhead $T$ of the pilot should be at least greater than the number of BS antennas $N$ i.e. $T>N$. This condition is undesired and unaffordable because huge number of antennas $N$ is used in mmWave massive MIMO which is discussed in the literature review section.

To cope with this problem, I propose an adaptive selecting-network (ASN) in addition to the proposed system which is shown in Figure 2.

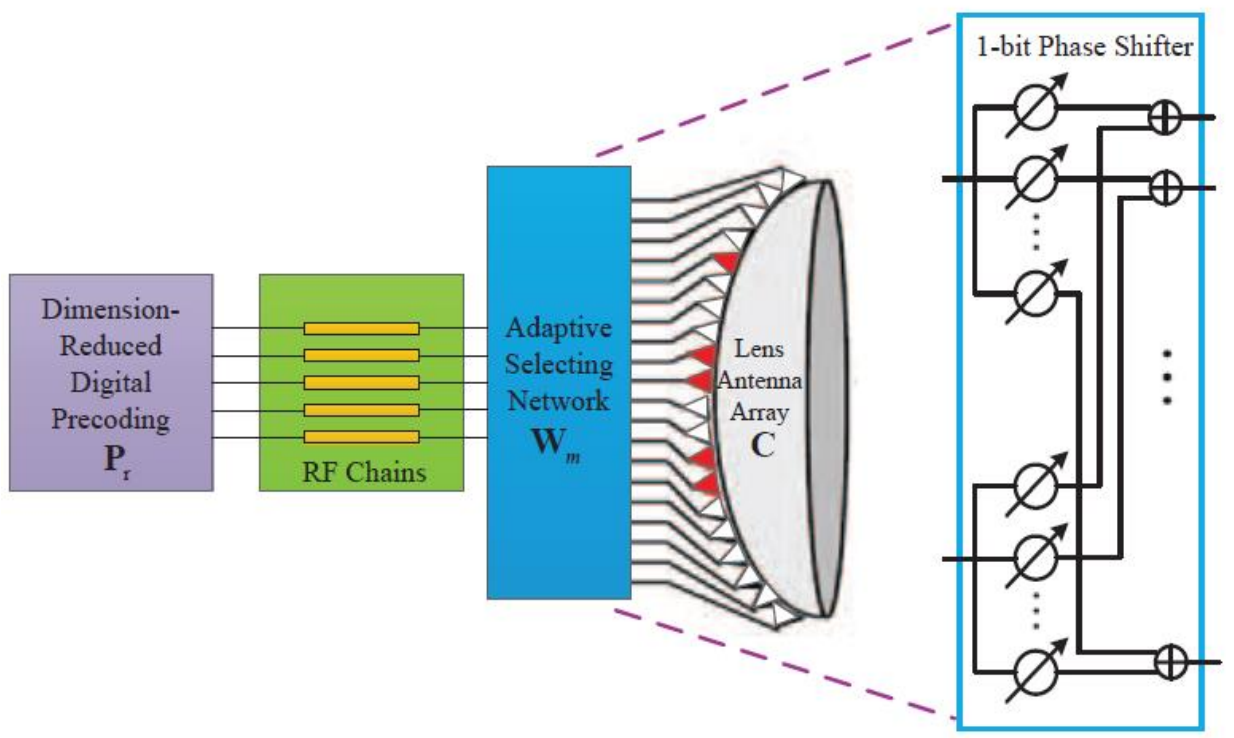

Fig.2. Proposed beamspace MIMO adaptive selective network.

The main difference between Figure 1(b) and Figure 2 systems is that the selective-network (SN) is substituted by the PSN. The ASN can be configured to perform the function of traditional beam-selection during data-transmission. Particularly, some PSs can be turned-off to perform the unselect operation whereas some PSs can be set to $0^{\circ}$ to perform the select operation of the concerned beam. This on-off phenomenon determines the beam-selection. Moreover, such ASN can also be used adaptively as analog-combiner $\mathrm{C}_{m}$ in 
order to combine the UL signals. The ASN can also assure that the measurement-matrix $\bar{y}_{k}$ in (11) can have the complete information of the beamspace-channel $\hat{h}_{k}$ and this holds true even if $T<N$ which is the desired objective. As discussed that there are limited scattering channel-paths in mmWave systems, so the beamspace channel exhibit sparse-structure. Thus, (11) can be deployed for the typical sparse-signal recovery issue. The next step of the proposed work is to design the analog-combiner $\bar{C}$ using the compressed-sensing (CS) theory. The mutual coherence for the $\bar{C}$ design is given by:

$$
\mu \triangleq \max _{i \neq j}\left|\bar{C}_{i}^{H} \bar{C}_{j}\right|
$$

Where $\bar{C}_{i}$ is the $i$ th-column of the analog combiner.

The mutual coherence $\mu$ should kept as small as possible during design. For small $\mu$, the Bernoulli randommatrix is used as the analog combiner $\bar{C}$ in this work. Which stated that each element of $\bar{C}$ is selected randomly with equal probability from $\frac{1}{\sqrt{T}}[-1,+1]$. This is suitable due the benefits that the same normalized-amplitude is shared by the entire element of the analog combiner, which can be practically realized by the PSs. The PSN resolution can be only 1 bit as the required phase-shifts are $0^{\circ}$ and $180^{\circ}$, which means that the energy and cost of the proposed PSN can be reduced substantially.

SD-based channel estimation:The measurement in (11) can be calculated by classical CS schemes such as compressive sampling matching pursuit (CoSaMP) and orthogonal matching pursuit (OMP). However, for the typical low-SNR case of mmWave massive MIMO system, which results due to the low-power transmission from the users and the lack of beamforming-gain, the beamspace channel $\hat{h}_{k}$ will devastated by noise. Consequently, the corresponding support of it will be inaccurately detected by the classical-CS schemes, which leads to degraded performance. To solve this problem, the support-detection (SD) algorithm is used to estimate the support of the beam space channel with high accuracy and better performance than the classical CSschemes. The two main bases of the SD-algorithm are obtained from two lemmas which are proved below that will be used for the proposed channel estimation.

Lemma-1: Any two beam space channel components are orthogonal asymptotically when the BS antennas in the proposed system tends to infinity. Using (2) the condition can be expressed as:

$$
\lim _{N \rightarrow \infty}\left|\hat{c}_{i}^{H} \hat{c}_{j}\right|=0
$$

This holds true for all $i, j=0,1,2,3, \ldots, L$ and $i \neq j$. This provides the clue that the entire problem of beamspace channel estimation can be decomposed into a series of sub-tasks problems that are independent from each other. Moreover, each of them will consider only one channel-component which is orthogonal to other components. Particularly, the strongest channel component is first estimated and then its effect is eliminated from the entire problem. In this way, the second strongest channel component is estimated. This method will be reiterated until the estimation of all channel components whose total length is $(\mathrm{L}+1)$ respectively.

Lemma-2: This gives the methodology of each channel component estimation in the beamspace. The same $i$ th-channel component $\hat{c}_{i}$ is considered which have $P_{T}$ as the total power and $P_{S}$ as the $S$-strongest-elements power of $\hat{c}_{i}$. Therefore, the ratio of the strongest-elements power and the total power of the channel-component $\hat{c}_{i}$ can be lower-bounded by the following condition:

$$
\frac{P_{S}}{P_{T}} \geq \frac{2}{N^{2}} \sum_{i=1}^{\frac{S}{2}} \frac{1}{\sin ^{2}\left[\frac{(2 i-1) \pi}{2 N}\right]}
$$


It means that once the strongest-elements position $p_{i}$ is obtained, the rest of the $S-1$ strongest-elements will be uniformly situated around it with $\frac{1}{N}$ interval. From this relationship, two important clues can be derived. First, the channel component cane be considered as sparse-vector. It is because most of the power is concentrated on a slight number of dominant-elements. So, only such small number of elements with strong power are considered and the others are discarded without any perceptible performance loss. Secondly, the sparse-vector support can be exclusively calculated by $p_{i}$ as follows:

$$
\operatorname{supp}\left(\hat{c}_{i}\right)=\bmod _{N}\left(p_{i}-\frac{\mathrm{v}}{2}, \ldots, p_{i}+\frac{\mathrm{v}-2}{2}\right)
$$

Where $\bmod _{N}($.$) is the modulo-operation with respect to N$ which assures the all entries in the support belong to the set $\{1,2,3, N\}$. To obtain the strongest-elements, the cardinality of the support is taken, which gives the number of strongest-elements present in the channel-component. The extracted $S$-strongest-elements provide the $S$-columns of the analog-combiner $\bar{C}$ in (11) and the nonzero-element the corresponding channelcomponent can be calculated using the classical least-square (LS) scheme. Figure 3 illustrates the normalizedamplitude distribution channel-components elements.

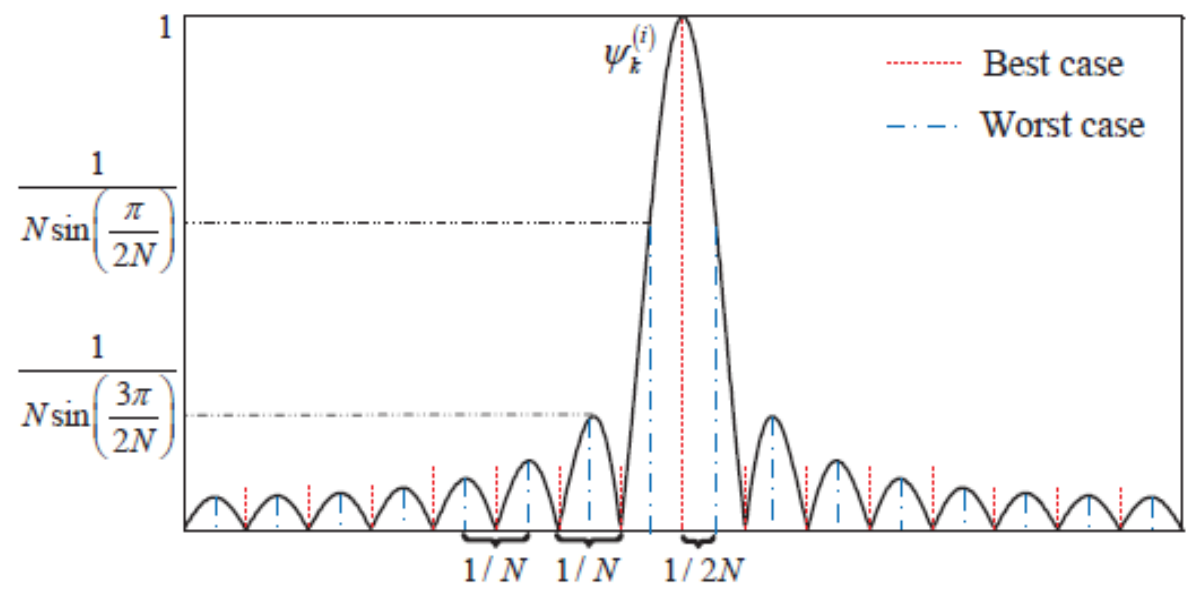

Fig.3. The normalized amplitude distribution of the channel components elements.

The flowchart in Figure 4 illustrates the step-by-step procedure of the proposed-SD algorithm for channel estimation. It is important to note that the main difference between the proposed SD-algorithm and the classical-CS algorithms is that the proposed scheme provide channel support-detection, whereas, the other schemes do not provide such beneficial parameter. Also, the proposed scheme provides only estimate the strongest-elements position whereas, the classical-CS locate the positions in an iterative and cyclic manner irrespective of the power-level of the channel-components which is undesired. 


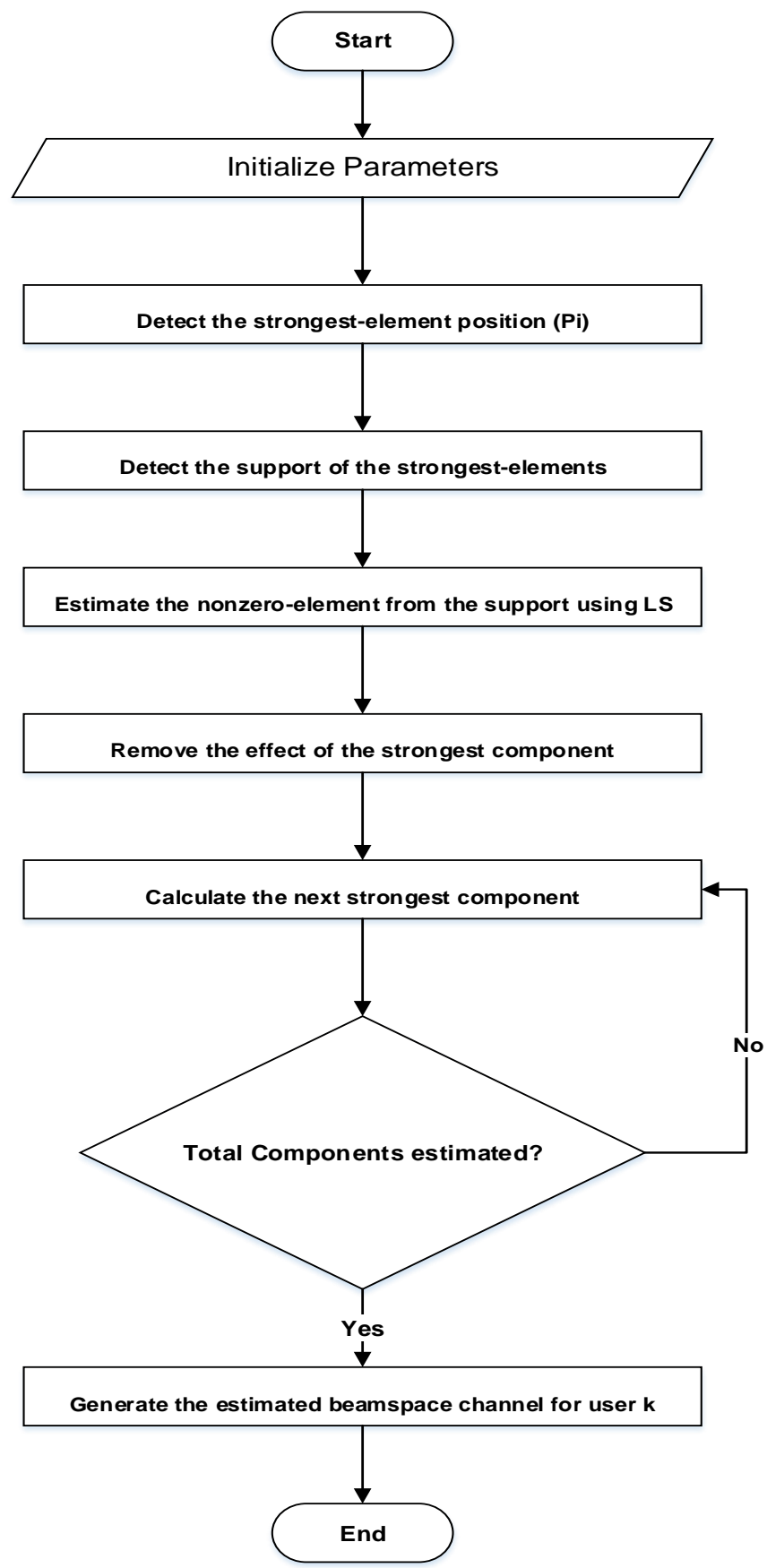

Fig.4. Proposed SD-algorithm for channel estimation.

The proposed scheme detects accurately the beamspace channel-support with higher probability as compare to the classical-CS schemes. It is pictorially compared in Figure 5. 


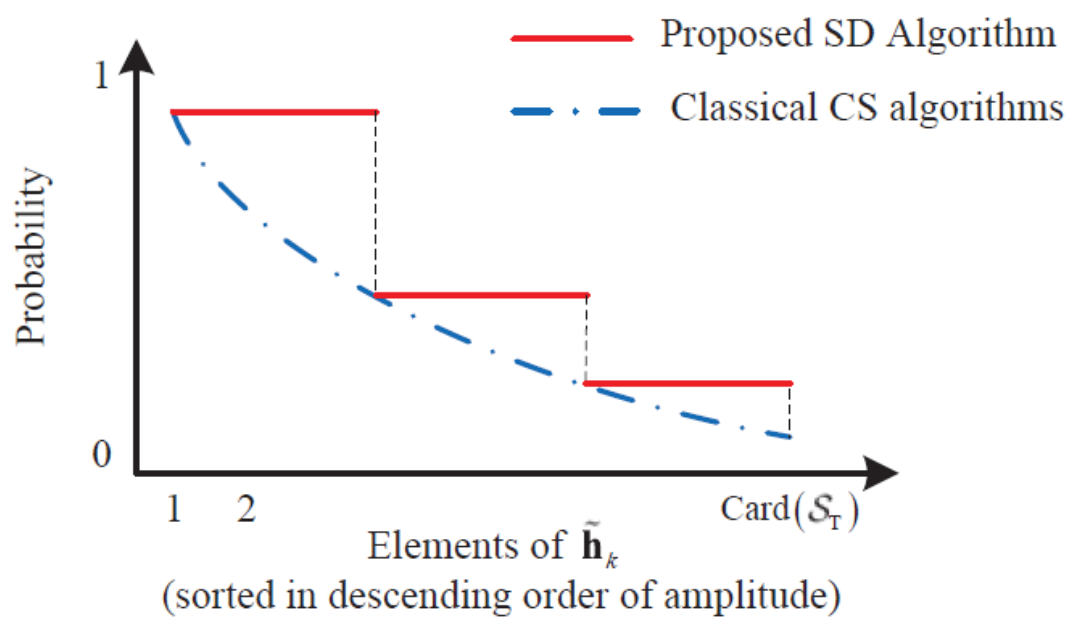

Fig.5. Probability comparison of the proposed SD and the classical-CS algorithms.

Table 1 shows the detail of the simulation parameters for the proposed system which are utilized for simulations results.

Table 1. Simulation Parameters

\begin{tabular}{lcc}
\hline Parameter & Symbol & Value \\
\hline Number of lens antennas (beams) at the BS & $\mathrm{N}$ & 260 \\
Number of users & $\mathrm{K}$ & 20 \\
Total time slots & $\mathrm{T}$ & 100 \\
Number of channel paths & $\mathrm{L}$ & 3 \\
Number of RF chains & $\mathrm{N}_{\mathrm{RF}}$ & 20 \\
Signal wavelength & $\lambda$ & 1 \\
Antenna element spacing & $\mathrm{d}$ & 0.5 \\
Total pilot overhead & $T$ & 100 \\
Total number of blocks & $M$ & 5 \\
Total number of strongest elements & $S$ & 8 \\
Sparsity level & $\mathrm{S}(\mathrm{L}+1)$ & 32 \\
Signal-to-Noise Ratio & $\mathrm{SNR}$ & $30 \mathrm{~dB}$ \\
\hline
\end{tabular}

\section{Simulation Results}

Simulation results are given to verify our analytical formulation. Figure 6 depicts the comparison of the normalize mean square error (NMSE) for the proposed support-detection (SD) algorithm and the classical CSOMP algorithm. It is clear from the figure that, the proposed SD-algorithm for channel estimation shows much better NMSE-performance than the classical CS-OMP algorithm. Moreover, the gap between both algorithm is larger at low SNR regime and it is evident that the proposed scheme main objective is to improve the mmWave performance at this low-SNR region (e.g. below $15 \mathrm{~dB}$ ). As the typical problem of mmWave system is such low-SNR before performing the beamforming, therefore the proposed SD-algorithm is well-suited for the 
mmWave massive MIMO systems. Figure 7 compares the achievable sum-rate performance of the proposed SD-algorithm with the classical CS based algorithms for different uplink SNR values. This illustrates the influence of the mentioned beamspace channel estimation schemes on beam-selection. The interference-aware (IA) algorithm for beam-selection is proposed as it can support the proposed condition of $N_{R F}=K$. It is clear from the figure that by deploying the proposed SD-algorithm along with the IA beam-selection method shows better performance than its counterparts. It is more dominant for SNR at the uplink is low.

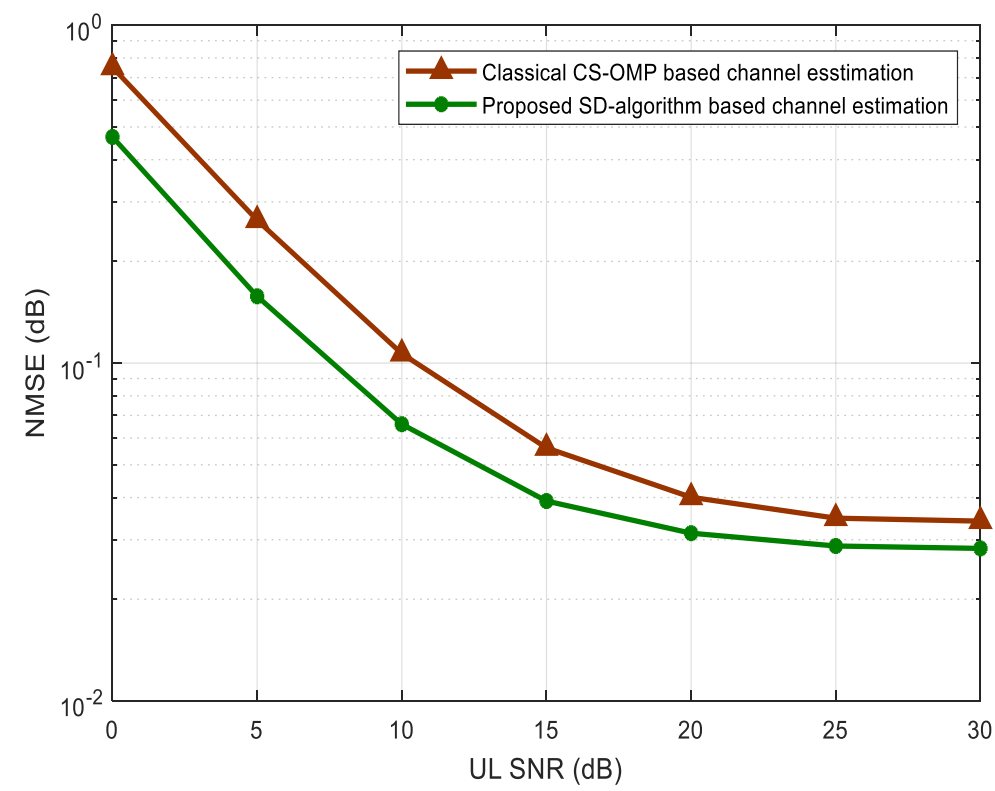

Fig.6. Comparison of the proposed SD with classical-CS algorithms in terms of NMSE.

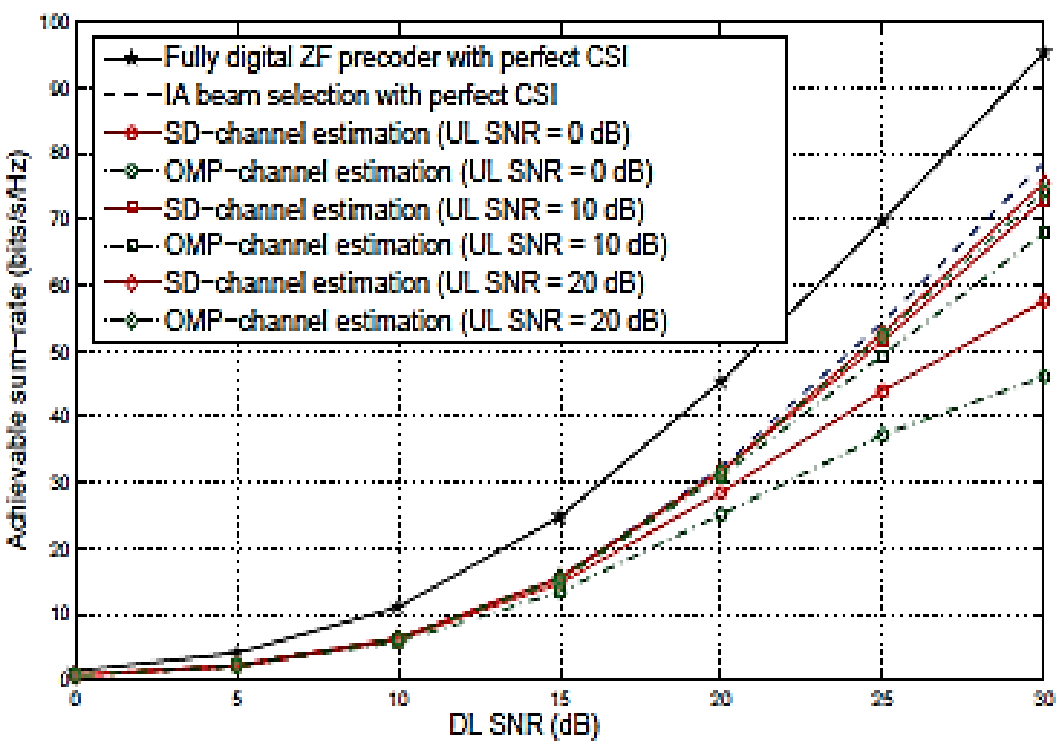

Fig.7. Comparison of the proposed SD with classical-CS algorithms in terms of achievable sum-rate. 
For average SNR level such as the 10dB case, the proposed SD-IA scheme shows close performance and this only requires 20 RF-chains whereas, the fully-digital zero-forcing (ZF) precoder with perfect channel state information (CSI) requires $260 \mathrm{RF}$-chains, which is big-difference to achieve the same performance level. Figure 8 illustrates the comparison between the classical conventional OMP scheme and the proposed ASDalgorithm for 3D beamspace channel estimation in terms of normalized mean square error against the SNR. It is clear that the proposed ASD-based 3D beamspace channel estimation shows improved performance in accuracy as compare to the conventional OMP algorithm. Furthermore, the proposed scheme performs better in low SNR region which makes it suitable for mmWave communications. As the total number time slots used is 256 in this model, whereas the total number of antenna elements are 1156. I can further conclude that the proposed ASD scheme reduces the pilot overhead and improves the overall system performance. Also, it reduces the required number of RF chains which is same as the number of users, not the number BS antennas, therefore, it significantly reduces the energy consumption, hardware complexity, cost per device and improves the energy efficiency of the mmWave massive MIMO systems.

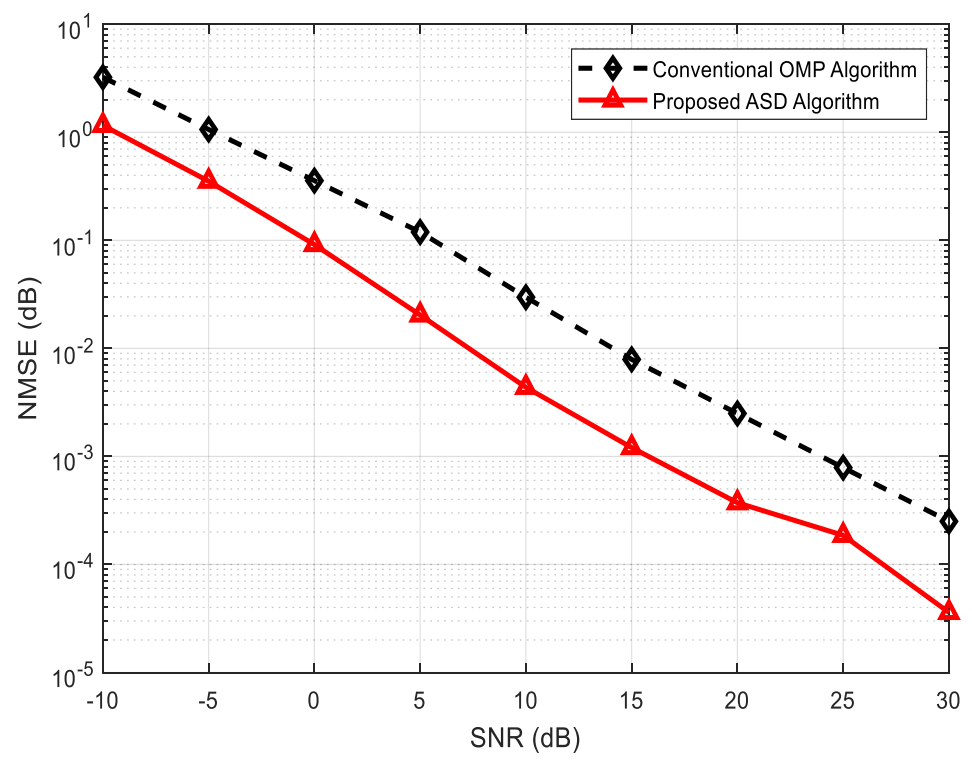

Fig.8. Comparison of the proposed ASD scheme and the conventional CS OMP scheme in terms of NMSE.

The sum rate performance evaluation is analysed in Figure 9 below. The ideal fully digital (FD) precoding scheme is used as benchmark for the proposed ASD scheme. It is clear from the Figure that, the proposed ASD with CSI IA-BS achieves near optimal sum rate performance with the limited number of RF chains, which makes it attractive from practical perspective. The ASD with CSI MM-BS also performs better which is below the IA-BS. This scheme improves the spectral efficiency even in low SNR. 


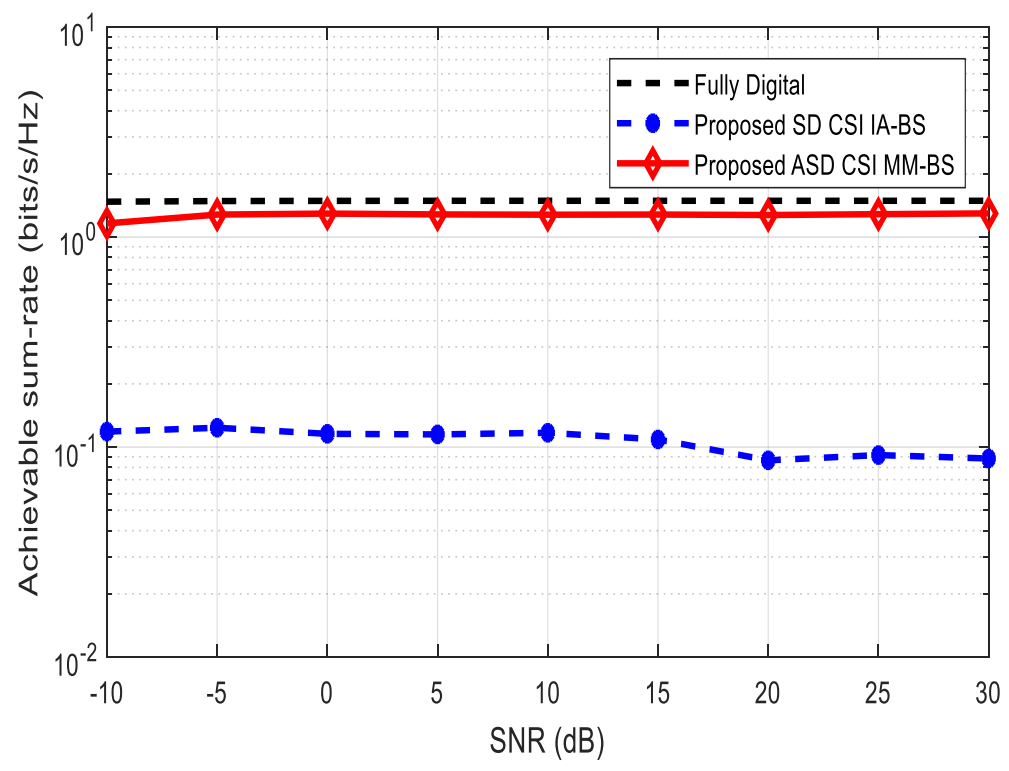

Fig.9. Sum rate comparison of the proposed ASD scheme with the fully digital precoding scheme.

\section{Conclusions}

This paper inspects the RF chain and pilot overhead issues of lens-based mmWave massive MIMO systems beamspace channel estimation. The main concern is to decrease the required number of RF chains and reduce the pilot-overhead via sparse characteristics of channel-vectors. Particularly, an adaptive selecting-network (ASN) is proposed which is low-cost to efficiently estimate the beamspace channel. Moreover, a supportdetection (SD) algorithm is proposed for channel estimation whose main principle is to use the sparse structural attributes of the beamspace channel and detect the support of the channel-component reliably. Simulation results reveal that the proposed SD-algorithm can attain much better performance in terms of NMSE and sumrate than the classical CS-based schemes. This work can be extended further easily by considering multipleantenna at the user-equipment (UE).

\section{References}

[1] R. W. Heath, N. Gonzalez-Prelcic, S. Rangan, W. Roh, A. Sayeed, "An overview of signal processing techniques for millimeter wave MIMO systems," IEEE Jr. Sel. Top. Signal Proc., vol. 10 (3), pp. 436-453, Apr. 2016.

[2] J. Brady, N. Behdad, and A. Sayeed, "Beamspace MIMO for millimeterwave communications: System architecture, modeling, analysis, and measurements," IEEE Tran. Anten. and Prop., vol. 61, (7), pp. 3814-3827, Jul. 2013.

[3] A. Alkhateeb, O. El Ayach, G. Leus, R. W. Heath, "Channel estimation and hybrid precoding for millimeter wave cellular systems," IEEE Jr. Sel. Top. Sig. Proc., vol. 8, (5), pp. 831-846, Oct. 2014.

[4] A. Alkhateeb, G. Leus, R. W. Heath, "Compressed sensing based multi user millimeter wave systems: How many measurements are needed?" in Proc. IEEE ICASSP, pp. 2909-2913, Apr. 2015,

[5] T. Kim and D. J. Love, "Virtual AoA and AoD estimation for sparse millimeter wave MIMO channels," in Proc. IEEE SPAWC Workshops, pp. 146-150, Jun. 2015. 
[6] Z. Pi, F. Khan, "An introduction to millimeter-wave mobile broadband systems", IEEE Comm. Mag., vol. 49, (6), pp. 101-107, Jun. 2011.

[7] Ericsson, "More than 50 billion connected devices", white paper: http://www.ericsson.com/res/docs/whitepapers/wp-50 billions.pdf

[8] M. R. Akdeniz, Y. Liu, M. K. Samimi, S. Sun, S. Rangan, T. S Rappaport, "Millimeter wave channel modeling and cellular capacity evaluation", IEEE Jr. Sel. Areas in Comm., Vol. 32, (6), Jun. 2016.

[9] S. Han, C. L, Z. Xu and C. Rowell, "Large-scale antenna systems with hybrid precoding analog and digital beamforming for millimeter wave 5G," IEEE Comm. Mag., vol. 53, issue no. 1, pp. 186-194, Jan. 2015.

[10] Y. Zeng, R. Zhang, "Millimeter wave MIMO with lens antenna array: A new path division multiplexing paradigm," IEEE Trans. On Comm., vol.64, issue no. 4, pp. 1557-1571, Apr. 2016.

[11] J. Brady, N. Behdad, and A. Sayeed, "Beamspace MIMO for millimeterwave communications: System architecture, modeling, analysis, and measurements," IEEE Trans. Ant. and Propag., vol. 61, no. 7, pp. 3814-3827, Jul. 2013.

[12] J. Brady, N. Behdad, and A. Sayeed, "Beamspace MIMO for millimeterwave communications: System architecture, modeling, analysis, and measurements," IEEE Trans. Ant. and Propag., vol. 61, no. 7, pp. 3814-3827, Jul. 2013.

[13] J. Hogan and A. Sayeed, "Beam selection for performance-complexity optimization in high-dimension MIMO systems," in Proc. CISS, Mar. 2016, pp. 337-342.

[14] L. Yang, Y. Zeng, and R. Zhang, "Efficient channel estimation for millimeter wave MIMO with limited RF chains," in Proc. IEEE ICC, May 2016, pp. 1-6.

[15] L. Dai, X. Gao, S. Han, C.-L. I, and X. Wang, "Beamspace channel estimation for millimeter-wave massive MIMO systems with lens antenna array," to appear in IEEE/CIC ICCC, Jul. 2016.

[16] Z. Xiao, T. He, P. Xia, and X.-G. Xia, "Hierarchical codebook design for beamforming training in millimeter-wave communication," IEEE Trans. Wireless Commun., vol. 15, no. 5, pp. 3380-3392, May 2016.

[17] S. Hur, T. Kim, D. J. Love, J. V. Krogmeier, T. A. Thomas, A. Ghosh, "Millimeter wave beamforming for wireless backhaul and access in small cell networks," IEEE Tran. Comm., vol. 61, (10), pp. 4391- 4403, Oct. 2013.

[18] D. Zhu, J. Choi, R.W. Heath, "Auxiliary beam pair enabled AoD and AoA estimation in mmWave FDMIMO systems,” IEEE Tran. Wire Commun., arXiv preprint arXiv:1610.05587, Oct. 2016.

[19] Z. Marzi, D. Ramasamy, U. Madhow, "Compressive channel estimation and tracking for large arrays in mm-wave picocells,” IEEE Jr. Sel. Top. Sig. Proc., vol. 10, (3), pp. 514-527, Apr. 2016.

[20] J. Lee, G. T. Gil, Y. H. Lee, "Channel estimation via orthogonal matching pursuit for hybrid MIMO systems in millimeter wave communications," IEEE Tran. Comm., vol. 64, (6), pp. 2370-2386, Jun. 2016.

[21] T. Kim, D. J. Love, "Virtual AoA and AoD estimation for sparse millimeter wave MIMO channels", IEEE SPAWC Workshops, Jun. pp. 146-150. 2015.

\section{Authors' Profiles}

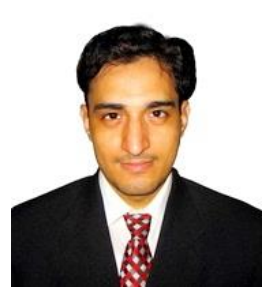

Imran Khan received his B.Sc. Degree in Electrical Engineering from the University of Engineering and Technology Peshawar, Pakistan in 2014 with Honors. He is currently working toward his M.Sc. Degree in Communication and Electronics Engineering from UET Peshawar. He is currently working as a Lecturer in the Department. His research interests include 5G, mmWave, Massive MIMO, IoT, WSN, Computer Networks and Wireless Communications. 
How to cite this paper: Imran Khan," Efficient Low-Overhead Channel Estimation for 5G Lens Based Millimeter-Wave Massive MIMO Systems", International Journal of Wireless and Microwave Technologies(IJWMT), Vol.8, No.3, pp. 42-57, 2018.DOI: 10.5815/ijwmt.2018.03.05 